\title{
Subcutaneous administration of tocilizumab is effective in myointimal hyperplasia remodelling in refractory Takayasu arteritis
}

\author{
A. Lo Gullo ${ }^{1 *}$, G. Mandraffino ${ }^{1 *}$, C.O. Aragona ${ }^{1}$, A. Molica Colella ${ }^{2}$, \\ A. Saitta ${ }^{1}$, E. Imbalzano ${ }^{1}$ \\ ${ }^{1}$ Department of Clinical and Experimental Medicine, University of Messina; \\ 2Unit of Rheumatology, Ospedale Papardo, Messina, Italy \\ *These authors contributed equally to this work.
}

\section{SUMMARY}

Takayasu arteritis (TA) is a chronic inflammatory disease of unknown origin that involves large and mediumsized arteries, primarily the aorta and its major branches. TA is a therapeutic challenge because corticosteroids and conventional immunosuppressive agents are not always effective or safe. Interleukin 6 (IL-6) has emerged as a key cytokine in the pathogenesis of TA and its serum levels have been shown to well correlate with disease activity.

We report a 19 years old female patient with TA refractory to conventional immunosuppressive agents, successfully treated with subcutaneous tocilizumab, a humanized monoclonal antibody against IL-6 receptor, in which ultrasonography (US) was used as imaging tool to follow up the patient. Currently, clinical indices of disease activity, inflammatory markers, carotid intima media thickness (cIMT) as well as carotid pulse wave velocity (cPWV) normalised, while the prednisone dosage has been tapered.

Tocilizumab appears to be a good option in refractory TA, with a remarkable steroid-sparing effect. In addition, it seems to have a favourable effect on endothelial function, as it improved cIMT and PWV.

Key words: Tocilizumab; intima media thickness; arterial stiffness; Takayasu arteritis; ultrasound.

Reumatismo, 2017; 69 (4): 184-188

\section{INTRODUCTION}

$\mathrm{T}$ akayasu arteritis (TA) is a chronic inflammatory vasculitis of unknown origin affecting large vessels, predominantly the aorta and its main branches (1). Vessel inflammation leads to wall thickening, fibrosis, stenosis, and thrombus formation (1). To date, no published controlled clinical trials are available, and the evidence on management strategies and therapeutic guidelines is mainly based on descriptive studies $(1,2)$. Despite the paucity of clinical reports, corticosteroids and immunosuppressant drugs are the mainstay of treatment, but relapses are common when the treatment is tapered (1); indeed, there is no consensus about the long term treatment of TA patients (3).
IL-6 is a pleiotropic cytokine which exerts powerful pro-inflammatory effects at a systemic level (1). The IL-6/s-IL-6 receptor (IL-6R) complex is likely to be a major player in the pathogenesis of large vessel vasculitis; moreover, IL-6 is highly expressed in the aortic tissue from patients with TA $(1,3)$, suggesting that IL-6 may contribute to the pathogenesis of TA.

Often, TA diagnosis is not established before the arterial stenosis occurs; computed tomography (CT) and magnetic resonance imaging (MRI) provide a reliable visualization of the vessel lumen and vessel wall inflammation (4). However, they may fail to adequately reveal signs of inflammation of distal vessels. In contrast, ultrasonography (US) provides a good overview of the affected arteries, including carotid, axil- 
lary, brachial, and femoral arteries with a significantly high resolution, and US displays a pathognomonic circumferential wall thickening in TA (4).

In the present study we report a sonographic follow-up of a patient affected by refractory TA treated with subcutaneous tocilizumab, a humanized monoclonal antibody against IL-6R. In this case, we observed a clinically significant improvement of vascular involvement and a persistent disease remission; US was confirmed as a useful complementary tool for evaluation of inflammatory and clinical activity (5).

\section{CASE REPORT}

From 2011 to 2015, five women were newly diagnosed with TA in our Internal Medicine Unit, University of Messina, Italy. The median age was 23 (range 12-43) and the mean disease duration was 5.9 years. All patients were on treatment with steroids (prednisone, mean dose $15 \mathrm{mg} /$ day) and methotrexate $10 \mathrm{mg} /$ week. During the follow up, the disease was well controlled with no evidence of disease progression on imaging in four patients. Indeed, one patient had a poorly controlled TA, with subsequent relapses and progression of disease at imaging evaluation. As a consequence, we decided to use TCZ for induction and maintenance of remission.

This 19-year-old female presented in January 2012 with myalgia, arthralgia, weakness, headache, and photopsia on the left side. On physical examination, she had erythema nodosum over the right leg, a left sided carotid bruit and normal blood pressure.

Erythrocyte sedimentation rate (ESR) was $20 \mathrm{~mm} / 1^{\text {st }} \mathrm{h}$ (normal values $<20 \mathrm{~mm} / 1^{\text {st }} \mathrm{h}$ ) and C-reactive protein (CRP) was $1.24 \mathrm{mg} /$ $\mathrm{dL}$ (normal values $<0.5 \mathrm{mg} / \mathrm{dL}$ ).

Carotid US examination showed a significant diffuse intima-media thickness (IMT) increase of $4.4 \mathrm{~mm}$ on the left common carotid artery (LCCA), determining a concentric $\sim 90 \%$ stenosis at the bifurcation. MRI confirmed US findings. According to EULAR recommendation, TA was diagnosed; consequently, prednisone $50 \mathrm{mg} /$ day and methotrexate $10 \mathrm{mg} /$ weekly were started (2).

In November 2012, the carotid US showed an improvement of the IMT $(3.3 \mathrm{~mm})$, with a decrease of stenosis (about $85 \%$ ); at this time, a vascular surgeon was consulted in order to verify the suitability of an operative approach. The vascular surgeon suggested continuing with medical therapy only, and prednisone dose was gradually tapered (up to $15 \mathrm{mg}$ per day) while methotrexate was increased to 12.5 $\mathrm{mg} /$ week, in order to spare corticosteroid administration.

In March 2013 she had a clinical relapse with carotidodynia; however, a new US examination showed a LCCA IMT of 3.1 $\mathrm{mm}$, inducing a $\sim 80 \%$ stenosis. ESR was $20 \mathrm{~mm} / 1^{\mathrm{st}} \mathrm{h}, \mathrm{CRP} 7.4 \mathrm{mg} / \mathrm{dL}$.

Positron emission CT (PET-CT) was performed, showing intense fluorodeoxyglucose uptake associated with intimal thickening of LCCA. Prednisone was increased to $50 \mathrm{mg} /$ day and methotrexate to $20 \mathrm{mg} /$ weekly.

Over the following fifteen months she was well, steroids were tapered up to $5 \mathrm{mg}$ /day and MTX to $15 \mathrm{mg} /$ weekly; laboratory results showed normal values of ESR and CRP, as well as blood cell count.

US examination was repeated in January 2014, and a further improvement of vascular status was observed (1.8 mm IMT on LCCA). Indeed, the right common carotid artery (RCCA) presented with a IMT $<0.6$ $\mathrm{mm}$ at every evaluation since the onset of the disease.

However, in October 2014, a routine US revealed an involvement of the right side: subclavian artery intima media hyperplasia, and a 75-80\% stenosis involving RCCA were found with a maximum carotid IMT of $7 \mathrm{~mm}$ (eccentric plaque). The examination also showed a stable carotid disease on the left side $(1.8 \mathrm{~mm}, \sim 75 \%$ concentric stenosis).

Prednisone was increased again to $50 \mathrm{mg} /$ day and methotrexate to $25 \mathrm{mg} /$ weekly. At the next evaluation (March 2015), US revealed a myointimal remodelling on RCCA: $45 \%$ stenosis and $4.1 \mathrm{~mm}$ IMT; LCCA presented with $75 \%$ stenosis $(2.0$ 
$\mathrm{mm}$ ); ESR was $39 \mathrm{~mm} / 1^{\mathrm{st}} \mathrm{h}$, CRP $1.7 \mathrm{mg} /$ $\mathrm{dL}$; prednisone was accordingly prescribed at a stable dose of $50 \mathrm{mg}$ per day.

Indian Takayasu Activity Score (ITAS) was 6, and Kerr index was 3; CRP was $1.09 \mathrm{mg} / \mathrm{dL}$, and ESR $35 \mathrm{~mm} / 1^{\text {st }} \mathrm{h}$. Carotid pulse wave velocity (PWV) was also assessed for the first time at this examination $(15.65 \mathrm{~m} / \mathrm{s})$.

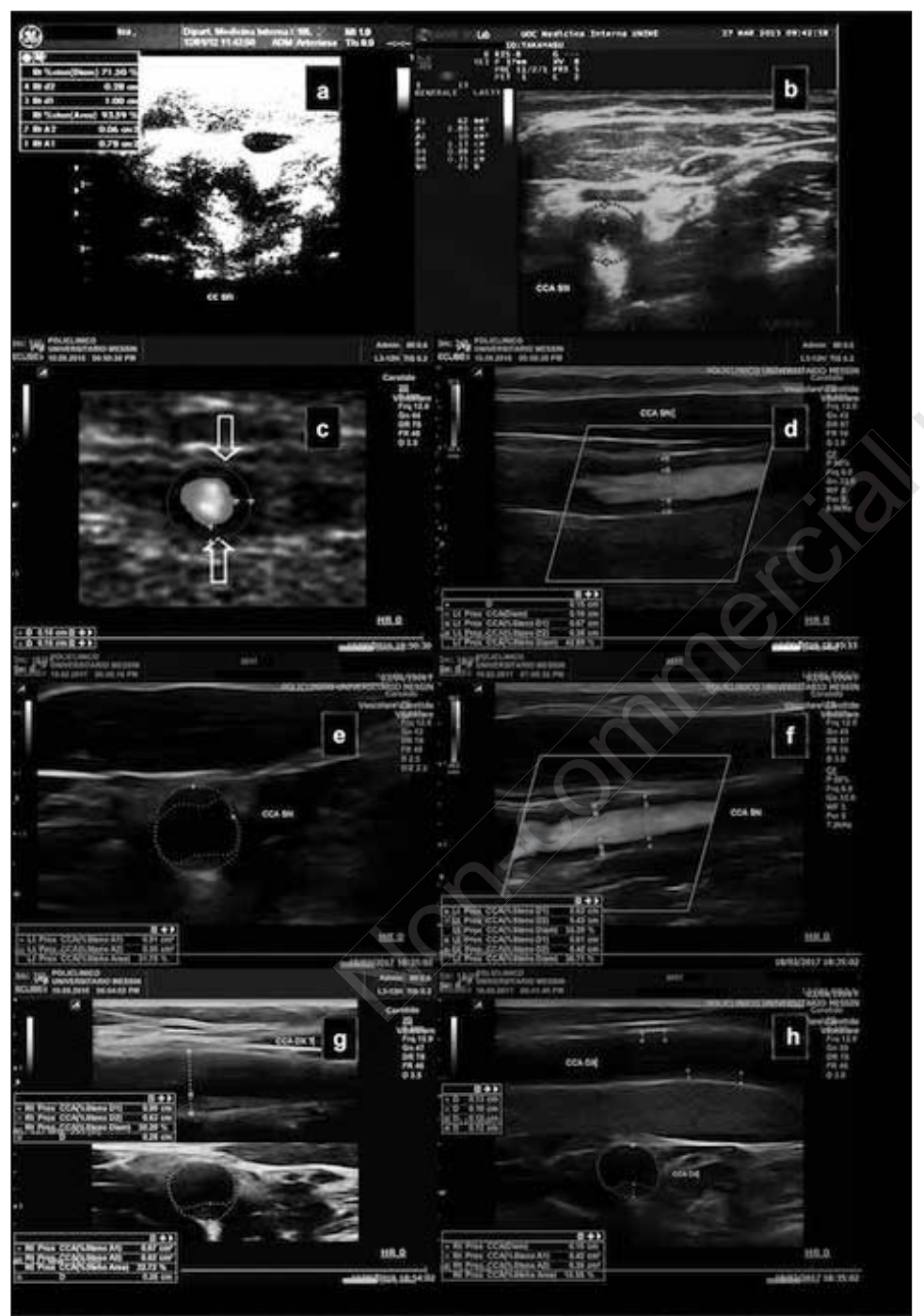

Figure 1 - Multipanel figure: US evaluation at different timepoints: a) Clinical onset (January, 2012); axial scan of LCCA; b) First re-evaluation (March, 2013); axial scan of LCCA; c) US re-evaluation (September, 2016); axial scan of LCCA; d) US re-evaluation (September, 2016); longitudinal scan of LCCA; e) US re-evaluation (February, 2017); axial scan of LCCA; f) US re-evaluation (February, 2017); longitudinal scan of LCCA; g) US re-evaluation (September, 2016); longitudinal (upper) and axial (lower) scan of RCCA; h) US re-evaluation (February, 2017); longitudinal (upper) and axial (lower) scan of RCCA.
After approval from the local ethical committee, TCZ was started at $162 \mathrm{mg}$ every week subcutaneously in July 2015; methotrexate was reduced at the dose of $15 \mathrm{mg}$ weekly, and prednisone was decreased to $10 \mathrm{mg}$ per day. After 6 months of therapy, inflammatory markers dropped to ESR 2 $\mathrm{mm} / 1^{\text {st }} \mathrm{h}$, CRP $0.3 \mathrm{mg} / \mathrm{dl}$. Kerr and ITAS indices decreased to 1 and 0 , respectively. US showed a marked improvement of right common carotid stenosis (20\%), and IMT was $1.6 \mathrm{~mm}$ (eccentric plaque); on the left side, the stenosis grade was $50 \%$, and IMT $1.45 \mathrm{~mm}$; an improvement of stenosis of the right subclavian artery was also reported. Prednisone was discontinued and methotrexate reduced to $7.5 \mathrm{mg}$ weekly.

In May 2016 no signs of clinical and instrumental relapse were reported, as left stenosis remained 50\%, (IMT $1.40 \mathrm{~mm}$ ), and the other previous findings were modified. Furthermore, the patient said she felt well, and clinical chemistry confirmed the inactivity of inflammatory disease. In September 2016, clinical and sonography evaluations confirmed the clinical, laboratory and instrumental stability of the disease. At the latest comprehensive evaluation (February 2017), we found a further improvement of the vascular involvement (both IMT and PWV; Figure 1), of clinical chemistry (Table I), and of clinical status, including coenesthesia.

\section{DISCUSSION AND CONCLUSIONS}

IL-6 has a close implication in large-vessel vasculitis $(3,6)$. IL-6 modulates activation, proliferation and differentiation of various $\mathrm{T}$ cell lines including CD8 T, Th17 and T-reg cells; furthermore, IL-6 stimulates terminal differentiation of $\mathrm{B}$ cells and improves survival of plasma cells. High plasma levels of IL-6 in patients with TA correlate very well with disease activity (6). Therefore, pharmacological blockade of IL-6 is suggested as a potential target in this kind of vasculitis (7).

Preliminary experience proposed that TCZ, a humanized anti-IL-6 receptor antibody, could be an option for refractory 
Table I - Synoptic table of main clinical reports.

\begin{tabular}{|l|c|c|c|c|c|c|c|c|c|c|c|c|c|}
\hline & $\mathbf{1 / 1 2}$ & $\mathbf{1 1 / 1 2}$ & $\mathbf{3 / 1 3}$ & $\mathbf{5 / 1 3}$ & $\mathbf{1 / 1 4}$ & $\mathbf{6 / 1 4}$ & $\mathbf{1 0 / 1 4}$ & $\mathbf{3 / 1 5}$ & $\mathbf{0 7 / 1 5}$ & $\mathbf{1 2 / 1 5}$ & $\mathbf{5 / 1 6}$ & $\mathbf{9 / 1 6}$ & $\mathbf{2 / 1 7}$ \\
\hline Creatinine mg/dL & 0.8 & - & 0.7 & 0.9 & 0.7 & 0.9 & - & 1 & 0.9 & 0.8 & 0.9 & 0.8 & 0.7 \\
\hline AST UI/I & 13 & - & 12 & 13 & 12 & 14 & - & 14 & 11 & 20 & 40 & 16 & 22 \\
\hline ALT UI/I & 22 & - & 14 & 21 & 10 & 11 & - & 14 & 16 & 40 & 92 & 22 & 32 \\
\hline Hb gr/dL & 13.3 & - & 12.4 & 12.9 & 11.6 & 11.8 & - & 12.1 & 11.5 & 13.5 & 13.8 & 13.7 & 14 \\
\hline WBC mmc & 8700 & - & 6500 & 8020 & 9540 & 7580 & - & 6900 & 9200 & 7950 & 6100 & 7800 & 7060 \\
\hline PLT mmc & 296 & - & 302 & 320 & 375 & 320 & - & 335 & 343 & 285 & 315 & 252 & 257 \\
\hline ESR mm/h & 20 & - & 20 & 5 & 30 & 35 & - & 39 & 35 & 2 & 5 & 2 & 2 \\
\hline CRP mg/dL & 1.24 & - & 7.4 & 1.0 & 0.89 & 0.92 & - & 1.72 & 1.09 & 0.5 & 0.21 & 0.8 & 0.17 \\
\hline Lc IMT mm & 4.4 & 3.3 & 3.1 & - & 1.8 & - & 1.8 & 2.0 & 1.8 & 1.45 & 1.45 & 1.5 & 1.4 \\
\hline Left stenosis \% & $\sim 90$ & $\sim 85$ & $\sim 80$ & - & 65 & - & 65 & 75 & $\sim 65$ & $\sim 50$ & $\sim 50$ & 40 & 30 \\
\hline Rc IMT mm & 0.4 & 0.6 & 0.6 & - & 0.6 & - & 7 & 4.1 & 4.5 & 1.6 & 1.40 & 2.8 & 1.5 \\
\hline Right stenosis \% & - & - & - & - & - & - & $70-75$ & $40-45$ & 50 & 20 & $15-20$ & $\sim 25$ & 15 \\
\hline PWV m/s & - & - & - & - & - & - & - & - & 15.6 & 9.77 & 8.34 & 7.2 & 6.7 \\
\hline Prednisone (mg/die) & 50 & 15 & 50 & 25 & 20 & 5 & 50 & 50 & 10 & - & - & - & - \\
\hline MTX & 10 & 12.5 & 20 & 20 & 20 & 15 & 25 & 25 & 15 & 7.5 & - & - & - \\
\hline TCZ & - & - & $\bigcirc$ & $\bigcirc$ & $\bigcirc$ & $\bigcirc$ & $\bigcirc$ & $\bigcirc$ & $\mathbf{0}$ & $\mathbf{0}$ & $\mathbf{0}$ & $\mathbf{0}$ & $\mathbf{0}$ \\
\hline
\end{tabular}

TA (7), with a steroid-sparing effectiveness and with a good clinical and laboratory response (3). Goel et al reported that 6 out of 10 patients treated with TCZ for 6 months remained radiologically stable, as evaluated by angiography or Doppler ultrasonography (8). Tombetti et al reported on a treatment with TCZ of 7 refractory patients, for a median of 14 months. Three out of them had a complete clinical response, and the imaging confirmed the evidence of stabilization/ improvement of arterial lesions after treatment with TCZ (9).

TA most commonly affects the left subclavian and common carotid arteries (10), which can be easily and quickly examined by ultrasound $(1,4)$. Also, in our case TA affected carotid and subclavian arteries that were routinely examined on each visit by US. Our study shows for the first time that subcutaneous TCZ could be a valid option in case of refractory vasculitis, since ultrasound is a good imaging tool to follow-up the patient and to manage the therapy.

In addition, we showed an improvement of pulse wave velocity, a marker of arterial stiffness. Arterial stiffening is a well-known biomarker of endothelial dysfunction and it is considered a valuable predictor for future cardiovascular events (11). It has been proposed that endothelial injury plays a crucial role in the pathogenesis of systemic vascu- litis (12). Endothelial damage in rheumatic disease can be counteracted by endothelial repair and angiogenesis $(13,14)$. Beyond its anti-inflammatory effect, including the reductions in C-reactive protein and fibrinogen levels, TCZ seems to be able to stimulate endothelial regeneration and to improve arterial stiffness (15); therefore, TCZ might also have a promising role in improving $\mathrm{CV}$ risk in patients with TA.

In conclusion, TCZ appears to be a promising drug in refractory disease with a reasonable steroid sparing effect and safety. Our findings need to be further confirmed by a larger case series, but these promising results encourage deeper evaluation of IMT and endothelial homeostasis associated with TCZ treatment in patients affected by TA.

Conflict of interest: the authors declare that they have no conflict of interest.

Ethical approval: all procedures performed in studies involving human participants were in accordance with the ethical standards of the institutional and/or national research committee and with the 1964 Helsinki declaration and its later amendments or comparable ethical standards.

Funding: this research received no specific grant from any funding agency in the public, commercial, or not-for-profit sectors. 
Informed consent: informed consent was obtained from patients included in the study.

\section{REFERENCES}

1. Mason JC. Takayasu arteritis - advances in diagnosis and management. Nat Rev Rheumatol. 2010; 6: 406-15.

2. Mukhtyar C, Guillevin L, Cid MC, et al. EULAR recommendations for the management of large vessel vasculitis. Ann Rheum Dis. 2009; 68: 318-23.

3. Muratore F, Pipitone N, Salvarani C. Standard and biological treatment in large vessel vasculitis: guidelines and current approaches. Expert Rev Clin Immunol. 2017: 1-16.

4. Schmidt WA. Ultrasound in vasculitis. Clin Exp Rheumatol. 2014; 32: S71-7.

5. Fan W, Zhu J, Li J, Zhang W et al. Ultrasound morphological changes in the carotid wall of Takayasu's arteritis: monitor of disease progression. Int Angiol. 2016; 35: 586-92.

6. Park MC, Lee SW, Park YB et al. Serum cytokine profiles and their correlations with disease activity in Takayasu's arteritis. Rheumatology (Oxford). 2006; 45: 545-8.

7. Salvarani C, Magnani L, Catanoso M, et al. Tocilizumab: a novel therapy for patients with large-vessel vasculitis. Rheumatology (Oxford). 2012; 51: 151-6.

8. Goel R, Danda D, Kumar S, et al. Rapid con- trol of disease activity by tocilizumab in 10 'difficult-to-treat' cases of Takayasu arteritis. Int J Rheum Dis. 2013; 16: 754-61.

9. Tombetti E, Franchini S, Papa M, et al. Treatment of refractory Takayasu arteritis with tocilizumab: 7 Italian patients from a single referral center. J Rheumatol. 2013; 40: 2047-51.

10. Grayson PC, Maksimowicz-McKinnon K, Clark TM, et al. Distribution of arterial lesions in Takayasu's arteritis and giant cell arteritis. Ann Rheum Dis. 2012; 71: 1329-34.

11. Ikdahl E, Rollefstad S, Wibetoe G, et al. Predictive value of arterial stiffness and subclinical carotid atherosclerosis for cardiovascular disease in patients with rheumatoid arthritis. J Rheumatol. 2016; 43: 1622-30.

12. Brogan PA, Shah V, Brachet C, et al. Endothelial and platelet microparticles in vasculitis of the young. Arthritis Rheum. 2004; 50: 927-36.

13. Lo Gullo A, Mandraffino G, Sardo MA, et al. Circulating progenitor cells in rheumatoid arthritis: association with inflammation and oxidative stress. Scand J Rheumatol. 2014; 43: 184-93.

14. Reynolds JA, Robertson AC, Bruce IN, et al. Improving cardiovascular outcomes in rheumatic diseases: therapeutic potential of circulating endothelial progenitor cells. Pharmacol Ther. 2014; 142: 231-43.

15. Patschan S, Nemirovsky K, Henze E, et al. Tocilizumab increases EPC regeneration in rheumatoid arthritis. Scand J Rheumatol. 2014; 43: 528-30. 